\title{
Constraint-Based Approach for Analysis of Hybrid Systems ${ }^{\star}$
}

\author{
Sumit Gulwani ${ }^{1}$ and Ashish Tiwari ${ }^{2}$ \\ ${ }^{1}$ Microsoft Research, Redmond, WA 98052 \\ sumitg@microsoft.com \\ ${ }^{2}$ SRI International, Menlo Park, CA 94025 \\ tiwari@csl.sri.com
}

\begin{abstract}
This paper presents a constraint-based technique for discovering a rich class of inductive invariants (boolean combinations of polynomial inequalities of bounded degree) for verification of hybrid systems. The key idea is to introduce a template for the unknown invariants and then translate the verification condition into an $\exists \forall$ constraint, where the template unknowns are existentially quantified and state variables are universally quantified. The verification condition for continuous dynamics encodes that the system does not exit the invariant set from any point on the boundary of the invariant set. The $\exists \forall$ constraint is transformed into $\exists$ constraint using Farkas lemma. The $\exists$ constraint is solved using a bit-vector decision procedure. We present preliminary experimental results that demonstrate the feasibility of our approach of solving the $\exists \forall$ constraints generated from models of real-world hybrid systems.
\end{abstract}

\section{Introduction}

The model checking problem seeks to determine if a given system satisfies a given property. For several interesting classes of systems (and properties), the model checking problem is theoretically intractable. As a result, techniques have been developed that are relatively complete for either verification or falsification. Predicate abstraction and abstract interpretation are examples of the former, while bounded model checking (BMC) is an example of the latter. An attractive feature of BMC is that it reduces the search for (bounded) falsification to a single constraint that can be solved using powerful satisfiability modulo theories (SMT) solvers. One analog of BMC for verification is $k$ induction. The other analog, which we pursue in this paper in the context of hybrid systems, is an approach based on using templates to search for inductive invariants.

Inductive invariants are at the core of any general approach for verification. In the case of hybrid systems, initial work on discovering inductive invariants was based on using iterative fixed-point computation based approaches like abstract

\footnotetext{
* Research supported in part by the National Science Foundation under grant CNS0720721 and by NASA under Grant NNX08AB95A.
}

A. Gupta and S. Malik (Eds.): CAV 2008, LNCS 5123, pp. 190-203, 2008.

(C) Springer-Verlag Berlin Heidelberg 2008 
interpretation or model checking [6, 11, 2, Recently, constraint-based approaches have been proposed that search for invariants of some given form by reducing the problem to constraint solving over the unknowns in the templates [22, 17]. Constraint-based techniques offer two main advantages over fixed-point computation based techniques. First, they are goal-directed and hence have the potential to be more efficient. Second, they do not require the use of widening heuristics that lead to an uncontrollable loss of precision in fixed-point based techniques. Furthermore, constraint-based techniques can search for "deep" invariants of a known form, whereas the other techniques are more suited for "simple" invariants of (relatively speaking) an unknown form. Since hybrid systems typically have "deep" invariants of (a small number of) known simple forms, constraint-based technique are quite appealing. Even though they have demonstrated some success in the form of discovering equational invariants [22] and conservatively discovering conjunctions of polynomial inequalities [17], constraint-based techniques have not yet achieved their full potential in verification of hybrid systems.

In this paper, we develop the constraint-based approach further and show that it can be applied to discovering a rich class of inductive invariants for verification of hybrid systems. In particular, our constraint-based technique can be used for discovering invariants that involve disjunctions of polynomial inequalities. One part of the challenge here is in formulating the inductiveness requirement - if $I$ holds in the current state $\boldsymbol{x}$ and there is a transition from $\boldsymbol{x}$ to $\boldsymbol{x}^{\prime}$, then $I$ holds in the state $\boldsymbol{x}^{\prime}$-for the continuous dynamics. The key insight here is that this requirement can be captured precisely as a universally quantified formula, just as it can be done for discrete transitions. In the continuous case, inductiveness is equivalent to requiring that the vector field points "inwards" on the "boundary" of the invariant set $I$.

The key steps of our constraint-based approach for verification are

(1) introduce a template for the unknown inductive invariant and express the verification conditions as satisfiability of a $\exists \forall$ formula over the reals, where the existential quantification is over the template variables and the universal quantification is over the state variables (Section [3);

(2) use a generalization of Farkas' Lemma to eliminate the $\forall$ quantifiers and convert the $\exists \forall$ formula to an $\exists$ formula (over the reals) (Section 4.1); and

(3) use the bit-vector theory in SMT solvers to search for solutions of the $\exists$ formula in a bounded range (Section 4.2).

We start by defining continuous dynamical systems and hybrid systems in Section 2. We then show that the problem of discovering invariants and verifying safety can be reduced to solving $\exists \forall$ constraints over the reals (Section 3). We present our approach for solving these constraints in Section 4. We present several nontrivial examples of continuous dynamical systems and hybrid systems that were successfully analyzed using our approach (Section 5 ). We compare with related work in Section 6 before concluding. 


\section{Continuous Dynamical and Hybrid Systems}

A continuous dynamical system is a tuple $\langle X$, Init, Inv, $f\rangle$ where $X$ is a finite set of variables interpreted over the reals $\mathbb{R}, \mathbf{X}=\mathbb{R}^{X}$ is the set of all valuations of the variables $X$, Init $\subseteq \mathbf{X}$ is the set of initial states, Inv $\subseteq \mathbf{X}$ is the state invariant, and $f: \mathbf{X} \mapsto \mathbf{X}$ is a vector field that specifies the continuous dynamics (as $\dot{\boldsymbol{x}}=$ $f(\boldsymbol{x}))$. We assume that $f$ satisfies the standard assumptions for existence and uniqueness of solutions to ordinary differential equations. The set Inv specifies the domain where the system is defined. The semantics of a continuous dynamical system are standard.

Example 1. Consider the following adaptive cruise controller where a car is following a leading car maintaining a safe distance [9, 19]. Let $d, v_{f}, v$, and $a$ respectively represent the gap between the two cars, the velocity of the leading car, and the velocity and acceleration of the rear car. The system dynamics are given by the following differential equations [19]:

$$
\dot{v}=a, \quad \dot{a}=-3 a-3\left(v-v_{f}\right)+(d-(v+10)), \quad \dot{d}=v_{f}-v, \quad v_{f}=a_{f}
$$

where $a_{f}$ is the acceleration of the leading car and an input in this model. Formally, we have a linear continuous dynamical system $\langle X$, Init, Inv, $f\rangle$ where $X=\left\{v, v_{f}, a, d, a_{f}\right\}, f$ is defined by the right-hand sides of the above differential equations, $\operatorname{Inv}=\left\{v \geq 0, v_{f} \geq 0,-2 \leq a \leq 5,-2 \leq a_{f} \leq 5\right\}$, Init $=\{d=$ $\left.5, v=v_{f}, a=0\right\}$. The invariant Inv captures the physical constraints that the cars do not move backwards and that the acceleration of the two cars is bounded from above and below. The initial states indicate when the above control law may be invoked. The problem is to verify that the rear car would never collide with the car in front, i.e., always $d>0$. We note that reachability is decidable for certain classes of linear dynamical systems [13, but this example does not fall in these decidable classes.

A hybrid system $\mathrm{HS}=(\mathbf{Q}, X$, Init, Inv, $t, f)$ consists of a finite set of modes $\mathbf{Q}$, a finite set $X$ of variables - that together define the state space $\mathbf{Q} \times \mathbf{X}:=\mathbf{Q} \times \mathbb{R}^{X}$ of the system - a mapping Init : $\mathbf{Q} \mapsto 2^{\mathbf{X}}$ that defines the initial states (in each mode), a mapping Inv : $\mathbf{Q} \mapsto 2^{\mathbf{X}}$ that defines the state invariant of each mode, a mapping $f: \mathbf{Q} \mapsto(\mathbf{X} \mapsto \mathbf{X})$ that specifies the continuous dynamics in each mode, and a mapping $t: \mathbf{Q} \times \mathbf{Q} \mapsto 2^{\mathbf{X}}$ that specifies the discrete transitions. Specifically, for any two modes $q, q^{\prime} \in \mathbf{Q}$, the system can jump from a state $(\mathbf{q}, \mathbf{x})$ to any state $\left(\mathbf{q}^{\prime}, \mathbf{x}\right)$ if $\mathbf{x} \in t\left(\mathbf{q}, \mathbf{q}^{\prime}\right)$. Note that, for simplicity of presentation, we are forcing the discrete transitions to have identity reset maps (that is, $\boldsymbol{x}$ is not updated), but our method works in the other case as well. Hence, $t\left(\mathbf{q}, \mathbf{q}^{\prime}\right)$ is just the guard, or switching condition, for going from mode $\mathbf{q}$ to mode $\mathbf{q}^{\prime}$. We assume that the semantics of hybrid systems and the set of reachable states are defined in the standard way, see [1].

Example 2. We consider a model of adaptive cruise control coupled with transmission from 25$]$. The hybrid system here is described by $\langle\mathbf{Q}, X$, Init, Inv, $t, f\rangle$ 
where $\mathbf{Q}:=\{1 s t, 2 n d, 3 r d, 4 t h\} \times\{c c, a c c\} \times\{$ normal, maxbrake, max acc $\}$ and $X:=\left\{d, v, v_{f}, a_{f}\right\}$. Thus, the hybrid system has 24 modes depending on the gear of the rear car (1st, 2nd, 3rd, 4th), its cruise control mode (regular cruise control $c c$, or adaptive cruise control $a c c$ ), and its mode of operation (normal, max-braking, or max-acceleration). The dynamics in the 24 modes of the adaptive cruise control model is defined as follows:

$$
\begin{aligned}
& \dot{d}=v_{f}-v, \text { in all modes } \\
& \dot{v}=-3.5, \text { in all maxbraking modes } \\
& \dot{v}=6-i, \text { in all max-acceleration and } i \text {-th gear modes } \\
& \dot{v}=0.9\left(v_{d e s}-v\right), \text { in all normal, regular cruise control (cc) modes, and } \\
& \dot{v}=-0.66 v+0.08 d-0.4+0.26 v_{f}, \text { in all normal, adaptive (acc) modes }
\end{aligned}
$$

where $v_{d e s}$ is a parameter set to the desired velocity in the cruise control mode. The set $\operatorname{Inv}(q)$ is the conjunction of all the following applicable facts:

$$
\begin{array}{ll}
-3.5 \geq-0.66 v+0.08 d-0.4+0.26 v_{f} & \text { maxbrake, acc, all gears } \\
-3.5 \leq-0.66 v+0.08 d-0.4+0.26 v_{f} \leq 6-i & \text { normal, acc, } i \text {-th gear } \\
-0.66 v+0.08 d-0.4+0.26 v_{f} \geq 6-i & \text { maxacc, acc, } i \text {-th gear } \\
-3.5 \geq 0.9\left(v_{\text {des }}-v\right) & \text { maxbrake, cc, all gears } \\
-3.5 \leq 0.9\left(v_{\text {des }}-v\right) \leq 6-i & \text { normal, cc, } i \text {-th gear } \\
0.9\left(v_{\text {des }}-v\right) \geq 6-i & \text { maxacc, cc, } i \text {-th gear } \\
0 \leq v_{f} \leq 60,-3.5 \leq a_{f} \leq 5, d \leq 40 & \text { acc } \\
d \geq 38 & \text { cc } \\
0 \leq v \leq 6.7 & \text { 1st gear } \\
6.7 \leq v \leq 14.2 & \text { 2nd gear } \\
14.2 \leq v \leq 29.8 & \text { 3rd gear } \\
29.8 \leq v \leq 60 & \text { 4th gear }
\end{array}
$$

All discrete transitions have identity reset maps (that is, the continuous variables do not change values on discrete transitions). The guard $t\left(q, q^{\prime}\right)$ of a discrete transition from mode $q$ to $q^{\prime}$ is given by $\operatorname{Inv}(q) \cap \operatorname{Inv}\left(q^{\prime}\right)$. For example, there is a transition from normal, acc, 1st-gear to normal, acc, 2nd-gear if $v=6.7 \wedge-3.5 \leq$ $-0.66 v+0.08 d-0.4+0.26 v_{f} \leq 5$. For more details, see $[25]$.

The notation $K[X]$ denotes the set of polynomials with coefficients in $K$ and variables in $X$. We use $\mathbb{Q}$ and $\mathbb{Z}\left(\mathbb{Z}^{+}\right)$to denote the set of rationals and (positive) integers respectively.

\section{Verification of Hybrid Systems}

Given a hybrid system $\mathrm{HS}=(\mathbf{Q}, X$, Init, Inv, $t, f)$, and a safety property $S$ : $\mathbf{Q} \rightarrow 2^{\mathbf{X}}$, the problem of hybrid system verification is to determine if the set of reachable states of the hybrid system in each mode $q \in \mathbf{Q}$ is a subset of $S(q)$.

The classical approach for solving the verification problem involves finding an inductive invariant map $I: \mathbf{Q} \rightarrow 2^{\mathbf{X}}$ such that the following constraints, referred to as the verification condition, hold for each mode $q \in \mathbf{Q}$. 
A1. (Initial Constraint) Init $(q) \subseteq I(q)$.

A2. (Transition Constraint) For all modes $q^{\prime} \in \mathbf{Q}, I(q) \cap t\left(q, q^{\prime}\right) \subseteq I\left(q^{\prime}\right)$.

A3. (Safety Constraint) $I(q) \subseteq S(q)$.

A4. (Inductive Constraint) If the system is in a state from the set $I(q) \cap \operatorname{Inv}(q)$, then it continues to be in the set $I(q)$, provided it also remains in $\operatorname{Inv}(q)$, at any time in the future as per the dynamics $f(q) 1$

In this section, we present a constraint-based technique for discovering an inductive invariant map that maps different modes to closed semi-algebraic invariants of the form $\bigwedge_{i} \bigvee_{j} p_{i j} \geq 0$, where $p_{i j} \in \mathbb{Q}[X]$ are polynomials of bounded degrees over $X$. We further assume that the initial conditions Init $(q)$, the safety conditions $S(q)$, and the transition conditions $t\left(q, q^{\prime}\right)$ are semi-algebraic, and that the flow $f$ is given by polynomials. This class of polynomial hybrid systems is very general and covers a wide variety of examples.

The key idea of our technique is to translate the verification condition into a $\exists \forall$ constraint over real variables. (Section 4 then describes how to solve such formulas using Farkas lemma.) This is achieved by choosing a template, $\mathcal{I}: \mathbf{Q} \mapsto 2^{\mathbf{U}, \mathbf{X}}$, for the inductive invariant $I$, where $U$ is a finite set of new template parameters and $\mathcal{I}(q):=\bigwedge_{i} \bigvee_{j} p_{i j}^{\prime} \geq 0$ with $p_{i j}^{\prime} \in \mathbb{Q}[U, X]$. The first three constraints in the verification condition can be easily translated into a $\exists \forall$ constraint over real variables by simply substituting the invariant template $\mathcal{I}(q)$ in place of $I(q)$ and replacing $\subseteq$ relation by $\Rightarrow$ relation. (This is because the existence of $I$ gets translated to existence of the unknown parameters $U$.) The challenge is to do this for the invariant constraint (A4). For that, we make use of continuity to obtain the following critical (necessary and sufficient) verification condition for continuous dynamical systems.

Proposition 1. Let $\langle X$, Init, Inv, $f\rangle$ be a continuous dynamical system and I be a set such that $\bigwedge_{i=1}^{n}\left(\bigvee_{j=1}^{m} p_{i j} \geq 0\right)$ is the conjunctive normal form (CNF) of Inv $\Rightarrow I$. The set I satisfies Constraint A4 for the continuous dynamical system iff for all $i \in\{1, \ldots, n\}$ and all non-empty subsets $J \subseteq\{1, \ldots, m\}$ :

$$
I(\boldsymbol{x}) \wedge \bigwedge_{j \in J}\left(p_{i j}(\boldsymbol{x})=0\right) \wedge \bigwedge_{j \notin J}\left(p_{i j}(\boldsymbol{x})<0\right) \wedge \operatorname{Inv}(\boldsymbol{x}) \Rightarrow \bigvee_{j \in J}\left(\frac{d p_{i j}(\boldsymbol{x})}{d t} \geq 0\right)
$$

Here $\frac{d p}{d t}$ denotes the time derivative of $p$, also called the Lie derivative of $p$, in the vector field defined by $f$; that is, $\frac{d p}{d t}:=\sum_{x \in X} \frac{\partial p}{\partial x} \frac{d x}{d t}:=\sum_{x \in X} \frac{\partial p}{\partial x} f_{x}$.

Proposition 1 essentially says that the vector field should point "inwards" on the boundary of the set $\overline{\operatorname{Inv}} \cup I$. The boundary of $\bigvee_{j=1}^{m} p_{i j} \geq 0$ is contained in the union (over all subsets $J$ ) of the sets $\bigwedge_{j \in J} p_{i j}=0 \wedge \bigwedge_{j \notin J} p_{i j}<0$. For each set in this disjoint union, we have a formula in Constraint A4 stating that the vector field is pointing inwards. For instance, consider the set $p_{1} \geq 0 \vee p_{2} \geq 0$. Choosing $J=\{1\}$, we get the boundary points $p_{1}=0 \wedge p_{2}<0$. On these boundary points, the vector field points inwards iff the Lie derivative, $\frac{d p_{1}}{d t}$, of $p_{1}$ is non-negative.

${ }^{1}$ If $I(q)$ is a positive invariant set [5], then it also satisfies our condition. In general, our condition is weaker than that of positive invariant sets. 


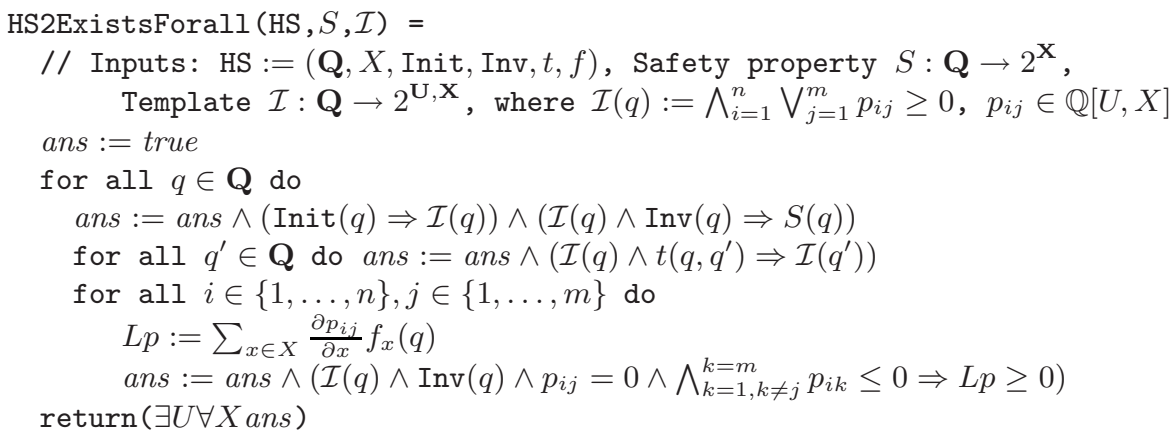

Fig. 1. Translating safety verification to satisfiability of an $\exists \forall$ formula

The Lie derivatives, $\frac{d p}{d t}$, in Equation A4' get reduced to polynomials as $\frac{d p}{d t}:=$ $\sum_{x \in X} \frac{\partial p}{\partial x} \frac{d x}{d t}$, and this summation simplifies into a polynomial if $p$ is a polynomial and $\frac{d \boldsymbol{x}}{d t}:=f(\boldsymbol{x})$ contains only polynomials. Since Constraints A1, A2, A3, and A4' are expressible in the first-order theory of reals, it follows from the decidability of this theory [26] that the problem of discovering bounded-size inductive invariants for polynomial hybrid systems over the class of positive boolean combination of (non-strict) polynomial inequalities of bounded degree is decidable. However, our interest is in obtaining a more practical technique for generating invariants. Figure 1 shows the construction of the $\exists \forall$ formula over real variables using the Constraints A1, A2, and A3, and Constraint A4, Since the number of constraints in A4 is exponential in $m$, Figure 1 uses a stronger variant of Constraint A4 that contains only a linear number of constraints, but that is usually sufficient in practice. In the next section we present our approach for solving these constraints.

Example 3 (Verification to $\exists \forall$ Constraint). Consider the dynamical system from Example 1 and the verification problem stated therein. Let us assume a template that searches for linear invariants:

$$
\mathcal{I}:=\left(\alpha v_{f}+\beta v+\gamma a+\delta d \geq \epsilon\right) \wedge(d \geq 0)
$$

where $U:=\{\alpha, \beta, \gamma, \delta, \epsilon\}$. Since Constraint A3 is trivially satisfied, the safety of the adaptive cruise control law reduces to the satisfiability of the following $\exists \forall$ constraint which essentially says that $\mathcal{I}$ is an inductive invariant.

$$
\begin{aligned}
\exists & U \forall X:\left(\left(d=5 \wedge v=v_{f} \wedge a=0 \Rightarrow \mathcal{I}\right)\right. \\
& \wedge\left(\mathcal{I} \wedge \operatorname{Inv} \wedge \alpha v_{f}+\beta v+\gamma a+\delta d=\epsilon \Rightarrow p \geq 0\right. \\
& \left.\wedge\left(\mathcal{I} \wedge \operatorname{Inv} \wedge d=0 \Rightarrow v_{f}-v \geq 0\right)\right)
\end{aligned}
$$

where $p$ is the Lie derivative of $\alpha v_{f}+\beta v+\gamma a+\delta d-\epsilon$ and is equal to $\alpha \dot{v}_{f}+\beta \dot{v}+$ $\gamma \dot{a}+\delta \dot{d}=\alpha a_{f}+\beta a-3 \gamma a-3 \gamma v+3 \gamma v_{f}+\gamma d-\gamma v-10 \gamma+\delta v_{f}-\delta v$. Similarly, $v_{f}-v$ is the Lie derivative of $d$. Note that $X$, Inv are defined in Example 1 


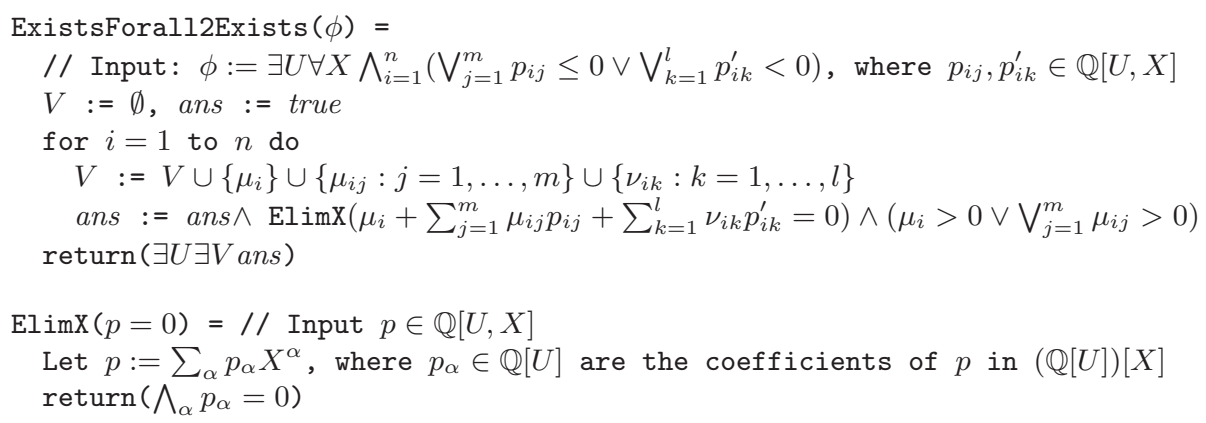

Fig. 2. Translating $\exists \forall$ formula to an $\exists$ formula

\section{Solving $\exists \forall$ Formulas}

We check for satisfiability of the $\exists \forall$ formula in two steps. First we eliminate the inner universal quantifier and next we check for satisfiability of the resulting existential formula over a finite domain using a satisfiability modulo theories (SMT) solver.

\subsection{Step 1: Eliminating Universal Quantification}

The inner universal quantifier from the $\exists \forall$ formula is eliminated using the following variant of Farkas Lemma.

Lemma 1. For polynomials $p_{j}, r_{k} \in \mathbb{Q}[X]$, the formula $\bigwedge_{j \in J} p_{j}>0 \wedge$ $\bigwedge_{k \in K} r_{k} \geq 0$ is unsatisfiable (over the reals) if there exist non-negative constants $\mu, \mu_{j}(j \in J)$, and $\nu_{k},(k \in K)$ such that $\mu+\sum_{j \in J} \mu_{j} p_{j}+\sum_{k \in K} \nu_{k} r_{k}=0$ and at least one of $\mu_{j}, \mu$ is strictly positive.

If polynomials $p_{j}, r_{k}$ are linear (more generally, linear only in the universal variables; for example, see the constraint in Example 3), then the condition above is both necessary and sufficient. However, the condition is not necessary for unsatisfiability when $p_{j}, r_{k}$ are arbitrary nonlinear polynomials 2 After applying Lemma 1, the universal variables can be eliminated by just equating the coefficients of each of the power products in the following expression to zero.

$$
\mu+\sum_{j \in J} \mu_{j} p_{j}+\sum_{k \in K} \nu_{k} r_{k}
$$

We can convert any universally quantified arithmetic formula $\forall X: \phi$ into an existentially quantified formula using the above lemma, as shown in Figure $2^{3}$ We illustrate this on our running example below.

2 There is a generalization of Farkas Lemma for arbitrary polynomials, called Positivstellensatz [15], obtained by replacing the multipliers $\mu_{j}, \nu_{k}$ by sum of squares of polynomials, but we did not use it in our experiments.

${ }^{3}$ This is achieved by converting $\phi$ into conjunctive normal form $\bigwedge_{i}\left(\bigvee_{j} p_{i j} \geq 0 \vee\right.$ $\left.\bigvee_{k} r_{i k}>0\right)$ and noting that $\forall X: \phi \equiv \bigwedge_{i} \forall X\left(\bigvee_{j} p_{i j} \geq 0 \vee \bigvee_{k} r_{i k}>0\right) \equiv$ $\bigwedge_{i}\left(\neg\left(\bigvee_{j} p_{i j} \geq 0 \vee \bigvee_{k} r_{i k}>0\right)\right.$ is unsatisfiable $) \equiv \bigwedge_{i}\left(\left(\bigwedge_{j}-p_{i j}>0 \wedge\right.\right.$ $\left.\bigwedge_{k}-r_{i k} \geq 0\right)$ is unsatisfiable). We can now use Lemma 1 on each conjunct. 
Example 4. Consider the $\exists \forall$ formula in Example3. To avoid clutter, we illustrate the $\forall$ elimination on a simpler subformula from that formula:

$$
\exists U \forall X:\left(\alpha v_{f}+\beta v+\gamma a+\delta d \geq \epsilon \wedge d=0 \wedge 2 a \geq-7 \Rightarrow v_{f}-v \geq 0\right)
$$

Using Lemma 1 the validity of the above formula is equivalent to the existence of constants $V:=\left\{\nu_{1}, \lambda, \nu_{2}, \mu_{1}, \mu_{2}\right\}$ such that

$$
\nu_{1}\left(\alpha v_{f}+\beta v+\gamma a+\delta d-\epsilon\right)+\lambda d+\nu_{2}(2 a+7)+\mu_{1}\left(v-v_{f}\right)+\mu_{2}=0,
$$

and $\nu_{1}, \mu_{1}, \mu_{2} \geq 0$ and at least one of the $\mu$ 's is strictly positive. By equating the coefficients to 0 , we get the following existentially quantified formula,

$$
\begin{aligned}
\exists U \exists V: \nu_{1} \alpha-\mu_{1}=0 & \wedge \nu_{1} \beta+\mu_{1}=0 \wedge \nu_{1} \gamma+2 \nu_{2}=0 \wedge \nu_{1} \delta+\lambda=0 \wedge \\
\mu_{2}-\nu_{1} \epsilon+7 \nu_{2}=0 & \wedge \bigwedge_{i} \mu_{i} \geq 0 \wedge \bigwedge_{i} \nu_{i} \geq 0 \wedge\left(\mu_{1}>0 \vee \mu_{2}>0\right)
\end{aligned}
$$

A possible solution is

$$
\nu_{1}=2, \lambda=-2, \nu_{2}=1, \mu_{1}=2, \mu_{2}=1, \alpha=1, \beta=-1, \gamma=-1, \delta=1, \epsilon=4 .
$$

Note that $\mu_{1}$ is strictly positive. This corresponds to the inductive invariant $v_{f}-v-a+d \geq 4$. We remark here that the full example contains additional constraints, but the above solution for $U$ continues to be a solution and it is the solution computed by our tool.

We now state the correctness of our approach as follows.

Theorem 1. Let HS be a hybrid system and $S$ a safety property. For any template $\mathcal{I}$, if the constraint ExistsForall2Exists(HS2ExistsForall(HS, $S, \mathcal{I})$ ) is satisfiable, then for every reachable state $(q, \boldsymbol{x})$ of $H S$, it is the case that $\boldsymbol{x} \in S(q)$.

\subsection{Step 2: Solving the $\exists$ Constraint Using an SMT Solver}

We have reduced the verification problem to the satisfiability of some (existentially quantified) nonlinear constraints. The important point to note here is that we are interested in finding solutions, rather than showing unsatisfiability, of the generated existential formula.

We search for solutions of the nonlinear constraints using the bit-vector decision procedure of an SMT solver. The translation of $\exists Y: \phi$ to bit-vectors is obtained in several steps. First polynomials in $\mathbb{Q}[Y]$ that occur in $\phi$ are converted to polynomials in $\mathbb{Z}[Y]$ by multiplying suitably by positive integer constants. Next we pick an integer lower bound $\boldsymbol{l}$ and an integer upper-bound $\boldsymbol{u}$ for the variables $Y$. Finally, we search for integer solutions for $Y$ in the chosen finite range by searching for the bit-level representation. We choose a size for the bitvectors by conservatively estimating the number of bits that would be required to evaluate the polynomials in $\phi$ over the range $\boldsymbol{l} \leq Y \leq \boldsymbol{u}$. The pseudo-code for the translator is given in Figure 3 . 


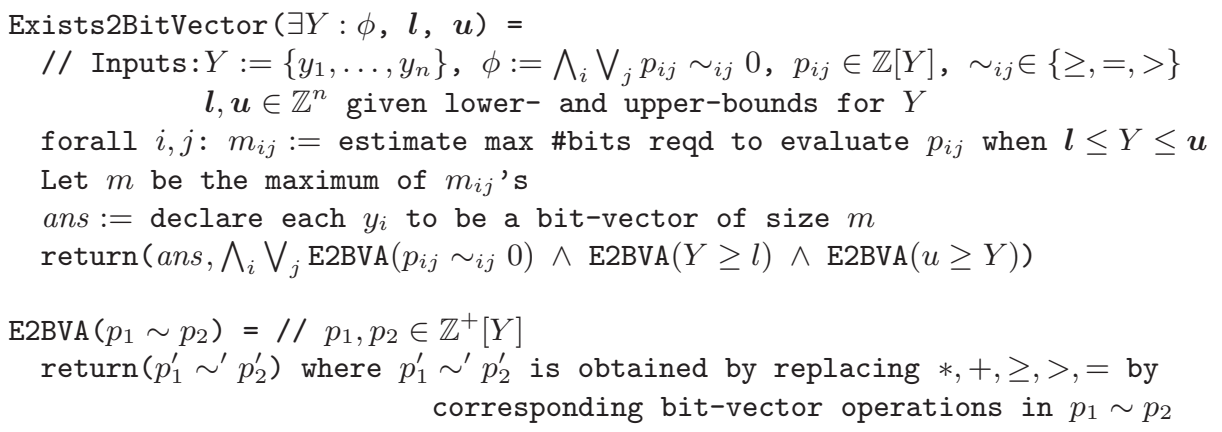

Fig. 3. Converting satisfiability checking to bit-vector satisfiability problem. The bitvector instance searches for all bounded integer solutions for $Y$ in the range $\boldsymbol{l} \leq Y \leq \boldsymbol{u}$ that satisfy $\phi$.

\subsection{Discussion}

Comparing the overall approach to bounded model checking, we note that both approaches translate the analysis problem into a constraint satisfiability problem. In the case of BMC, the generated constraint encodes existence of a counterexample, whereas here the generated constraint encodes existence of a proof.

When verifying a large hybrid system, we start by applying our technique to a small component of the system using linear and quadratic templates. If we find an invariant for the smaller subsystem, we use it as a starting point to construct refined templates for the full system (Example 6).

Our constraint-based technique for verification can be used for solving instances of the synthesis problem as well. The technique uniformly treats the entities of the verification condition, which includes both the inductive invariants and the description of the system. It does not matter whether the invariants are unknown or parts of the system are unknown or both of them are unknown. As long as there is sufficient information in the system description, the constraintbased approach can potentially find a solution for the unknown quantities.

Example 5. Consider the classical thermostat example, which is a hybrid system with two modes: in the "on" mode, temperature $x$ increases as $d x / d t=100-x$, and in the "off" mode, it decreases as $d x / d t=-x$. We want to synthesize the control logic that determines when to switch modes. Assume initially mode is "off" and $x=78$. The goal is to ensure $75 \leq x \leq 80$ always. For simplicity, assume that the specified safety property, $75 \leq x \leq 80$, is also an inductive invariant (and we do not guess a template for the invariant). Assume that we guess that the guard for the transition from heater-on to heater-off mode is of the form $x \geq \alpha$ and that the guard for the reverse transition is $x \leq \beta$. We can now write the verification conditions as follows:

$$
\begin{aligned}
& \exists \alpha, \beta: \forall x:(x=75 \wedge x>\beta \Rightarrow-x \geq 0) \wedge(x=80 \wedge x>\beta \Rightarrow-x \leq 0) \wedge \\
& \quad(x=75 \wedge x<\alpha \Rightarrow 100-x \geq 0) \wedge(x=80 \wedge x<\alpha \Rightarrow 100-x \leq 0) \wedge \\
& \quad(x=78 \Rightarrow x>\beta)
\end{aligned}
$$


One solution returned by our constraint solver was $\alpha=\beta=76$. However, this solution leads to zeno behavior. We can add additional constraints (not described in this paper) that capture the requirement that the switching logic be most liberal, in which case we get $\beta=75$ and $\alpha=79$.

\section{Experimental Results}

The approach described in this paper has been partially implemented in the form of two separate components. The first component takes an $\exists \forall$ formula (over arbitrary nonlinear polynomials) and returns an $\exists$ formula. The second component takes the $\exists$ formula and creates a Yices [8] formula over bit-vectors. The implementation is in Lisp. The bit-vector decision procedure of Yices is used to finally search for solutions. The front-end step of generating the $\exists \forall$ formula from a hybrid system description has not been automated yet.

All examples presented in this paper were analyzed automatically using the above tools. Some results are reported in Table 1.

Example 6 (Adaptive Cruise Control with Transmission). Consider the cruise control model from Example 2. The safety property to establish is that intervehicle separation remains positive; specifically, $d \geq 5$. We assume that initially the rear car is in the mode normal, acc, 4-th gear satisfying $v=v_{f} \wedge-3.5 \leq$ $-0.66 v+0.08 d-0.4+0.26 v_{f} \leq 2 \wedge 29.8 \leq v \leq 60$. We want to prove the safety property assuming that the velocity $v_{f}$ of the leading car remains bounded between $30 \leq v_{f} \leq 60$.

Our tools prove the safety by generating the following invariant for each of the acc modes:

\begin{tabular}{l|l} 
Invariant & Modes \\
\hline $2 d-2 v+v_{f}-2 \geq 0, d \geq 5$ & normal, acc, all gears \\
$-350 \leq-66 v+8 d-40+26 v_{f}$ & normal, acc, all gears \\
false & max-braking, acc, all gears \\
$2 d-2 v+v_{f}-2 \geq 0, d \geq 5$ & max-acceleration, acc, all gears \\
\hline
\end{tabular}

Note that the max-braking mode is not reachable from the chosen initial states. We did not generate the invariants for all modes in one step. We first generated invariants for single modes and that gave us an idea of the form of invariants and helped refine our template. Using a refined template, we generated invariants for all the acc-modes simultaneously.

Example 7 (Human Blood Glucose Metabolism). We consider the model of insulin metabolism in the body of a Type-I diabetic patient 24, 14. For purposes of modeling insulin concentration in the human body, the body is divided into six compartments - brain $(B)$, heart $(H)$, gut $(G)$, lungs $(L)$, kidney $(K)$, and periphery $(\mathrm{P})$ - and each state variable represents the insulin concentration in one such compartment (there are two variables for the "periphery" compartment). 
Table 1. Experimental Results. We report the number of continuous variables (Dim) in the example, the size of the Yices formulas generated by the example in terms of the number of variables (Vars), the size of bit-vectors (Bits), and number of assertions (Assertions), and the time (Time) taken by Yices to find a model on a 64-bit Pentium $3.4 \mathrm{GHz}$ cpu with $2 \mathrm{MB}$ cache.

\begin{tabular}{|l|c|c|c|c|c|}
\hline Example & Dim & Vars & Bits & Assertions & Time \\
\hline \hline disjunction Ex. 9 & 2 & 14 & 6 & 50 & $7 \mathrm{~ms}$ \\
\hline delta-notch & 4 & 34 & 8 & 120 & $30 \mathrm{~ms}$ \\
\hline plankton Ex. 8] & 3 & 31 & 8 & 110 & $56 \mathrm{~ms}$ \\
\hline thermostat & 1 & 29 & 20 & 126 & $.45 \mathrm{~s}$ \\
\hline thermostat synthesis Ex. [5 & 1 & 21 & 20 & 75 & $1.2 \mathrm{~s}$ \\
\hline ACC Ex. 1 & 5 & 28 & 12 & 95 & $1.3 \mathrm{~s}$ \\
\hline acc-transmission Ex. 2 & 4 & 35 & 24 & 122 & $4.7 \mathrm{~s}$ \\
\hline insulin Ex. 7 & 7 & 66 & 18 & 180 & $18 \mathrm{~s}$ \\
\hline
\end{tabular}

The dynamics of the system are given as follows, see also [24]:

$$
\begin{aligned}
d I_{B} / d t & =-45 / 26 I_{B}+45 / 26 I_{H} \\
d I_{H} / d t & =45 / 99 I_{B}-312 / 99 I_{H}+90 / 99 I_{L}+72 / 99 I_{K}+105 / 99 I_{P V}+u \\
d I_{G} / d t & =72 / 94 I_{H}-72 / 94 I_{G} \\
d I_{L} / d t & =18 / 114 I_{H}-720 / 10000 I_{H}+72 / 118 I_{G}-2880 / 10000 I_{G}-90 / 118 I_{L} \\
d I_{K} / d t & =72 / 51 I_{H}-72 / 51 I_{K}-2160 / 10000 I_{K} \\
d I_{P V} / d t & =105 / 74 I_{H}-105 / 74 I_{P V}-674 / 1480 I_{P V}+674 / 1480 I_{P I} \\
d I_{P I} / d t & =1 / 20 I_{P V}-1 / 20 I_{P I}-21231 / 51580 I_{P I}
\end{aligned}
$$

The control input $u$ in this case is the insulin injected into the body by an external insulin pump. Since we assume a Type-I diabetic, there is no pancreatic insulin release and hence no feedback from the glucose metabolism model. Assuming that the input $u$ is bounded between 20 and 25, we can compute bounds or ranges for insulin concentrations in different body compartments. As remarked earlier, we can easily invert the analysis and ask for acceptable bounds on insulin injection rate that will ensure bounded insulin concentration levels in the body.

Example 8. Consider the following Phytoplankton Growth Model (see [3] and references therein): $\dot{x}_{1}=1-x_{1}-\frac{x_{1} x_{2}}{4}, \dot{x}_{2}=\left(2 x_{3}-1\right) x_{2}, \dot{x}_{3}=\frac{x_{1}}{4}-2 x_{3}^{2}$, where $x_{1}$ denotes the substrate, $x_{2}$ the phytoplankton biomass, and $x_{3}$ the intracellular nutrient per biomass. For this nonlinear dynamical system, we can immediately generate the following invariant: $0 \leq x_{1} \leq 2,0 \leq x_{2} \leq 1,0 \leq x_{3} \leq 1 / 2$.

Example 9 (Disjunctive Invariants). Our technique can be used to generate disjunctive invariants. Consider the system $d x / d t=-y, d y / d t=-x$ with initial states given by $x \geq 3$. Using the template $x \geq \alpha \vee y \geq \beta$, we can generate the invariant $x \geq 0 \vee y \geq 0$. 


\section{Related Work}

The approach of using templates and generating invariants of a specific form for hybrid systems was introduced simultaneously by Sankaranarayanan et. al. 22, and Prajna et. al. 16, 17, 18. In all such approaches, an $\exists \forall$ formula is generated, although this may not be explicitly stated. The various approaches differ in the form of the invariants considered, the technique used to generate the $\exists \forall$ formula, and the approach for solving it. Templates are restricted to polynomial equations in 22 and Proposition 1 is not required there. The approach for solving the $\exists \forall$ constraints is based on Gröbner basis computation. Polynomial inequality templates are used in [17, but a much weaker variant of Proposition 1 is used there. The constraint solving method in [17] is based on convex optimization and sum-of-squares computation and is, in essence, a slightly more general form of Lemma 1 inspired by Positivstellensatz. We build upon these works and explore a more precise translation into $\exists \forall$ constraints and the use of SMT solvers as the backend engine.

Tiwari [27] generated linear inductive invariants for linear systems. RodriguezCarbonell and Tiwari 20] showed that the best (strongest) possible polynomial equational invariant was computable for hybrid systems with linear dynamics in each mode. Pappas et al. have also considered the problem of computing invariants, but only for linear systems, using interesting techniques [28, 29].

In software program analysis, constraint based techniques have been successfully applied for discovering linear arithmetic invariants [7, 21, 23, 10], non-linear polynomial invariants [12] and invariants in the combined theory of linear arithmetic and uninterpreted functions [4].

\section{Conclusion}

The verification technique based on guessing the form of inductive invariant and searching for invariants of that form using SMT solvers is a potent approach for verifying hybrid systems. Its extension to solving the synthesis problem is left for future work. Using efficient nonlinear constraint solvers directly could also significantly improve the performance of our approach and remains to be explored.

\section{References}

[1] Alur, R., Courcoubetis, C., Halbwachs, N., Henzinger, T.A., Ho, P.-H., Nicollin, X., Olivero, A., Sifakis, J., Yovine, S.: The algorithmic analysis of hybrid systems. Theoretical Computer Science 138(3), 3-34 (1995)

[2] Alur, R., Henzinger, T., Lafferriere, G., Pappas, G.J.: Discrete abstractions of hybrid systems. Proceedings of the IEEE 88(2), 971-984 (2000)

[3] Bernard, O., Gouze, J.-L.: Global qualitative description of a class of nonlinear dynamical systems. Artificial Intelligence 136, 29-59 (2002) 
[4] Beyer, D., Henzinger, T., Majumdar, R., Rybalchenko, A.: Invariant Synthesis for Combined Theories. In: Cook, B., Podelski, A. (eds.) VMCAI 2007. LNCS, vol. 4349, pp. 378-394. Springer, Heidelberg (2007)

[5] Blanchini, F.: Set invariance in control. Automatica 35, 1747-1767 (1999)

[6] Chutinan, A., Krogh, B.H.: Verification of Polyhedral-Invariant Hybrid Automata Using Polygonal Flow Pipe Approximations. In: Vaandrager, F.W., van Schuppen, J.H. (eds.) HSCC 1999. LNCS, vol. 1569. Springer, Heidelberg (1999)

[7] Colón, M., Sankaranarayanan, S., Sipma, H.: Linear Invariant Generation Using Non-linear Constraint Solving. In: Hunt Jr., W.A., Somenzi, F. (eds.) CAV 2003. LNCS, vol. 2725, pp. 420-432. Springer, Heidelberg (2003)

[8] Dutertre, B., de Moura, L.: A fast linear-arithmetic solver for dpll(t). In: Ball, T., Jones, R.B. (eds.) CAV 2006. LNCS, vol. 4144, pp. 81-94. Springer, Heidelberg (2006), http://yices.csl.sri.com/

[9] Godbole, D., Lygeros, J.: Longitudinal control of the lead car of a platoon. IEEE Transactions on Vehicular Technology 43(4), 1125-1135 (1994)

[10] Gulwani, S., Srivastava, S., Venkatesan, R.: Program analysis as constraint solving. In: Proc. PLDI (2008)

[11] Henzinger, T.A., Ho, P.-H., Wong-Toi, H.: HyTech: A model checker for hybrid systems. Software Tools for Technology Transfer 1, 110-122 (1997)

[12] Kapur, D.: Automatically generating loop invariants using quantifier elimination. In: Deduction and Applications (2005)

[13] Lafferriere, G., Pappas, G.J., Yovine, S.: Symbolic reachability computations for families of linear vector fields. J. Symbolic Computation 32(3), 231-253 (2001)

[14] Parker, R.S., Doyle, F.J., Peppas, N.A.: A model-based algorithm for blood glucose control in type I diabetes patients. IEEE Trans BioMed Eng. 46(2) (1999)

[15] Parrilo, P.A.: Structured semidefinite programs and semialgebraic geometric methods in robustness and optimization. PhD thesis, California Inst. of Tech. (2000)

[16] Prajna, S.: Barrier certificates for nonlinear model validation. In: Proc. IEEE Conference on Decision and Control (2003)

[17] Prajna, S., Jadbabaie, A.: Safety verification of hybrid systems using barrier certificates. In: Alur, R., Pappas, G.J. (eds.) HSCC 2004. LNCS, vol. 2993, pp. 477-492. Springer, Heidelberg (2004)

[18] Prajna, S., Jadbabaie, A., Pappas, G.: A framework for worst-case and stochastic safety verification using barrier certificates. IEEE Trans. Aut. Control (2005)

[19] Puri, A., Varaiya, P.: Driving safely in smart cars. In: Proceedings of the 1995 American Control Conference (1995)

[20] Rodriguez-Carbonell, E., Tiwari, A.: Generating polynomial invariants for hybrid systems. In: Morari, M., Thiele, L. (eds.) HSCC 2005. LNCS, vol. 3414, pp. 590605. Springer, Heidelberg (2005)

[21] Sankaranarayanan, S., Sipma, H., Manna, Z.: Non-linear loop invariant generation using gröbner bases. In: POPL 2004 (2004)

[22] Sankaranarayanan, S., Sipma, H., Manna, Z.: Constructing invariants for hybrid systems. In: Alur, R., Pappas, G.J. (eds.) HSCC 2004. LNCS, vol. 2993, pp. 539 554. Springer, Heidelberg (2004)

[23] Sankaranarayanan, S., Sipma, H.B., Manna, Z.: Scalable Analysis of Linear Systems Using Mathematical Programming. In: Cousot, R. (ed.) VMCAI 2005. LNCS, vol. 3385, pp. 25-41. Springer, Heidelberg (2005)

[24] Sorensen, J.T.: A physiologic model of glucose metabolism in man and its use to design and assess improved insulin therapies for diabetes. PhD thesis, Dept. Chem. Eng., Massachusetts Inst. Technology (MIT), Cambridge (1985) 
[25] Stursberg, O., Fehnker, A., Han, Z., Krogh, B.H.: Verification of a cruise control system using counterexample-guided search. Control Engineering Practice 12(10), 1269-1278 (2004)

[26] Tarski, A.: A Decision Method for Elementary Algebra and Geometry, 2nd edn. University of California Press (1948)

[27] Tiwari, A.: Approximate reachability for linear systems. In: Maler, O., Pnueli, A. (eds.) HSCC 2003. LNCS, vol. 2623, pp. 514-525. Springer, Heidelberg (2003)

[28] Yazarel, H., Pappas, G.J.: Geometric programming relaxations for linear system reachability. In: Proc. 2004 American Control Conference (2004)

[29] Yazarel, H., Prajna, S., Pappas, G.J.: S.O.S. for safety. In: Proc. 43rd IEEE Conference on Decision and Control (2004) 